\title{
A passive guidance system for a robotic walking assistant using brakes
}

\author{
Daniele Fontanelli ${ }^{1}$ and Antonio Giannitrapani ${ }^{2}$ and Luigi Palopoli ${ }^{3}$ and Domenico Prattichizzo ${ }^{2}$
}

\begin{abstract}
Robotic walkers are a promising techological direction to improve the mobility of older adults. In this paper, we propose a guidance mechanism based on the differential actions of two brakes located on the rear wheels. The guidance problem can be seen as a complex dynamic path following problem, with limited control authority in the actuators and with important constraints on the user comfort. We cast the problem into an optimisation framework and propose a computationally efficient solution that can be implemented on low cost devices with limited computing power. Simulation results are presented to validate the proposed approach.
\end{abstract}

\section{INTRODUCTION}

Although mobility and physical activity are key to improve quality of life of older adults, various physical and cognitive disabilities can impede senior citizens' exercise [1], [2]. Robotic walking assistants can alleviate this problem, by providing physical and psychological support to the assisted person (AP) in her navigation of complex environments. Several walking aids have been proposed over the years, most of them exploiting the paradigm of passive robotics in order to guarantee the safety of the user [3]. One of the first commendable contribution is the Cobot, a cane with a controlled caster wheel that guides the user to a desired path [4]. Different proposals of this kind range from steeringonly controlled walkers [5], to fully actuated assistive carts [6] (see, e.g., [7] for a recent overview of robotic walking assistants). A further step toward an improved safety is the outright removal of driving motors, which are replaced by electromagnetic brakes, as first proposed in [8] and later on developed in [9]. The resulting passive walker can be guided by a differential control on the brakes, using the same idea adopted by the automotive industry for stability control (ESP). Along the same line is the work [10], which proposes a brake controller guaranteeing the dissipative nature of the overall system.

In this paper, we propose a mechanical guidance system for older adults, based on a standard walker pushed by the AP and controlled through suitable braking actions. Our work differs from previous solutions in two important points.

This paper has received funding from the European Unions Horizon 2020 Research and Innovation Programme - Societal Challenge 1 (DG CON$\mathrm{NECT} / \mathrm{H}$ ) under grant agreement $\mathrm{n}^{\circ} 643644$ "ACANTO - A CyberphysicAl social NeTwOrk using robot friends".

D. Fontanelli is with the Department of Industrial Engineering (DII), University of Trento, Via Sommarive 5, Trento, Italy daniele.fontanellidunitn.it. A. Giannitrapani and D. Prattichizzo are with the Department of Information Engineering and Mathematical Sciences, University of Siena, Via Roma 56, Siena, Italy \{giannitrapani, prattichizzo\}edii.unisi.it. L. Palopoli is with the Department of Information Engineering and Computer Science (DISI), University of Trento, Via Sommarive 5, Trento, Italy palopoli@disi.unitn.it
First, rather than forcing the user to move on a predefined trajectory, the purpose of the developed guidance system is to gently "suggest" to the AP the optimal direction of motion with a minimal impact on her comfort. The AP remains in charge of the final decision on the direction to take and more authoritative actions are taken by the system to avoid dangerous situations. This is achieved by defining a safety tunnel around the desired path and imposing corrective actions only when the walker approaches or crosses the tunnel boundaries. Second, we aim for a low cost solution that uses embedded hardware and avoids expensive force sensors. The problem of identifying a minimally intrusive braking action that keeps the user close to a planned route has been first formulated in our previous work [11]. The additional contributions of this work are: 1. a closed form solution for the optimisation problem that is implementable with minimal CPU requirements, 2 . the ability for the system to operate in the absence of a direct measurement of the forces applied by the AP to the walker, 3. a couple of heuristic solutions that allow the system to operate even when the optimisation problem has no feasible solutions.

The paper is organized as follows. Section II presents the dynamic model of the system, and recalls a control law for the path following problem with full actuation. The problem for the braking guidance system is formulated in Section III and its closed form solution is presented in Section IV. Numerical simulations are reported in Section V, whereas some conclusions are drawn in Section VI.

\section{BACKGROUND MATERIAL}

\section{A. System Overview}

A picture of the FriWalk, the intelligent walker currently under development within the "ACANTO" project [12], is visible in Fig. 1. The system is endowed with sensors and embedded computing boards that empower it with cognitive abilities. The walker is derived from a standard commercial product. The rear wheels are instrumented with electromechanical brakes and by position encoders. It can sense the surrounding environment, predict the motion of human agents present in the scene a plan a safe course that avoids accidents or stressful conditions [13]. The path is "suggested" to the user mainly through a mechanical guidance support, complemented by audio/visual signals (conveyed through a touchscreen) and by tactile stimula, that are conveyed through an haptic device [14], [15].

\section{B. Model}

With reference to Fig. 2, let $\mathbf{x}=[x, y, \theta]^{T} \in \mathbb{R}^{2} \times S$ be the kinematic configuration of the cart, where $(x, y)$ are 


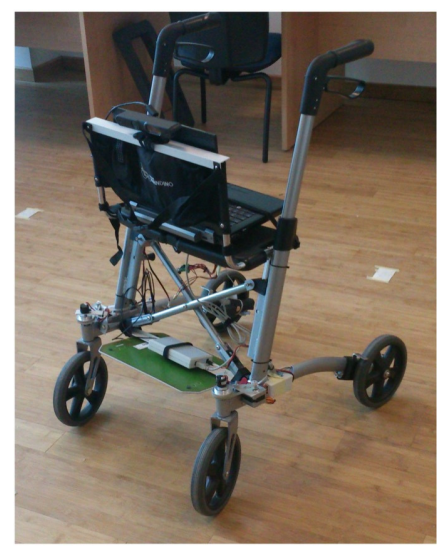

Fig. 1. Initial prototype of the FriWalk smart walker.

the coordinates of the mid-point of the rear wheel axle and $\theta$ is the orientation of the vehicle w.r.t. the $X_{w}$ axis. The dynamics of the considered walker can be described by the unicycle model

$$
\begin{array}{ll}
\dot{x}=v \cos (\theta), & \dot{v}=\frac{F}{m}, \\
\dot{y}=v \sin (\theta), & \dot{\omega}=\frac{N}{J}, \\
\dot{\theta}=\omega . &
\end{array}
$$

where $v$ is the forward velocity of the vehicle and $\omega$ its angular velocity. The control inputs are the external force $F$ acting on the vehicle along the direction of motion and the external torque $N$ about the $Z_{w}$-axis. The mass $m$ and moment of inertia $J$ of the cart are assumed to be known. Letting $d$ be the wheel axle length and $r$ the radius of the wheels, the force $F$ and torque $N$ can be expressed in terms of the the right wheel torque $\tau_{r}$ and left wheel torque $\tau_{l}$ through the following linear relationship

$$
F=\frac{\tau_{r}+\tau_{l}}{r} \text { and } N=\frac{\left(\tau_{r}-\tau_{l}\right) d}{2 r} .
$$

\section{Dynamic Path Following Problem}

A dynamic path planning problem consists in finding a control law for the force $F$ and torque $N$ in (1) such that the vehicle converges on a desired path. We are interested in solving a relaxed version of such a problem, since the objective is just to keep the cart "in proximity" of a desired path. To this aim, we follow a two steps approach: 1. compute a "desired" left and right wheel torque profile assuming full control authority, 2. approximate the desired torques by suitably modulating the left and right brakes. Since in the "ACANTO" framework the desired path is the result of an on-board planning algorithm, we can safely assume that such a path is smooth and that the path curvature is known (typically, the planner yields a path made of segments and circular arcs). Moreover, we assume that: a) the vehicle-to-path localization is solved (for instance, using visual apparatuses or other localization mechanisms) and b) a Frenet frame moving along the path is available. Let $(p, l)$ be the coordinate of the walker in the Frenet frame and denote

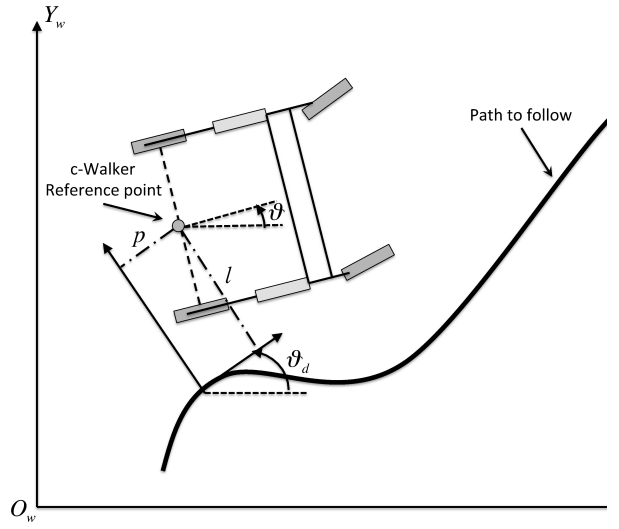

Fig. 2. Reference frames.

by $\theta_{d}$ the angle between the $X_{w}$-axis and the $X$-axis of the Frenet frame (see Figure 2). By letting $s$ be the curvilinear abscissa along the path and defining $\tilde{\theta}=\theta-\theta_{d}$, the vehicle dynamics (1) can be rewritten as (see [16]):

$$
\begin{array}{ll}
\dot{p}=-\dot{s}(1-c(s) l)+v \cos (\tilde{\theta}), \dot{v}=\frac{F}{m}, \\
\dot{l}=-c(s) \dot{s} p+v \sin (\tilde{\theta}), & \dot{\tilde{\omega}}=\dot{\omega}-c(s) \ddot{s}-c(s)^{\prime} \dot{s}^{2}, \\
\dot{\tilde{\theta}}=\tilde{\omega}, &
\end{array}
$$

where $c(s)$ is the path curvature defined as $c(s)=d \theta_{d}(s) / d s$, while $c^{\prime}(s)=d c(s) / d s$. The velocity $\dot{s}$ is a degree of freedom of the control law. Substituting (2) into (1), the rightmost equations of (3) we can be rewritten as

$$
\dot{v}=a_{f} u_{f}, \quad \dot{\tilde{\omega}}=a_{n} u_{n}-c(s) \ddot{s}-c(s)^{\prime} \dot{s}^{2},
$$

where $a_{f}=\frac{1}{r m}$ and $a_{n}=\frac{d}{2 r J}$ are two constant parameters, and

$$
u_{f}=\tau_{r}+\tau_{l}, \quad u_{n}=\tau_{r}-\tau_{l},
$$

represent the new control inputs. In [16] a solution to the dynamic path following problem has been provided using Lyapunov and backstepping techniques. Using a similar approach, we define the following Lyapunov control function

$$
\begin{aligned}
V_{d}= & \frac{1}{2}\left(k_{6}\left(p^{2}+l^{2}\right)+k_{1}(\tilde{\theta}-\delta(l, v))^{2}+\right. \\
& \left.+k_{7}\left(\omega-\omega_{d}\right)^{2}+k_{8}\left(v-v_{d}\right)^{2}\right),
\end{aligned}
$$

and the corresponding control law

$$
\begin{aligned}
\dot{s}= & v \cos (\tilde{\theta})+k_{2} p, \\
\delta(l, v)= & -k_{\delta} \tanh (l v), \\
\omega_{d}= & c(s) \dot{s}+\dot{\delta}(l, v)-\frac{k_{6}}{k_{1}} l v \frac{\sin (\tilde{\theta})-\sin (\delta(l, v))}{\tilde{\theta}-\delta(l, v)} \\
& -k_{3}(\tilde{\theta}-\delta(l, v)), \\
u_{f}= & \frac{1}{a_{f}}\left(\dot{v}_{d}-k_{5}\left(v-v_{d}\right)\right), \\
u_{n}= & \frac{1}{a_{n}}\left(\dot{\omega}_{d}-\frac{k_{1}}{k_{7}}(\tilde{\theta}-\delta(l, v))-k_{4}\left(\omega-\omega_{d}\right)\right) .
\end{aligned}
$$

In (7), $v_{d}$ is the desired forward velocity of the vehicle, $k_{i}>$ $0, i=1, \ldots, 8$, are the parameters of the controller and 
$k_{\delta} \in[3 \pi / 8, \pi]$ represents the maximum value of the $\delta(l, v)$ angle tracking. With such a choice, control law (7) makes $p$, $l, \tilde{\theta}$ and $v-v_{d}$ tend asymptotically to zero, i.e. the vehicle asymptotically converges to the desired trajectory. The reader is referred to [16] for further details.

\section{Dynamic of the Wheel}

In the following, the symbol $q_{(\cdot)}$ is adopted to refer indifferently to the quantities $q_{l}$ and $q_{r}$ related to the left and right side of the trolley, respectively. It is convenient to consider the half-car model, which allows us to analyse separately the forces acting on the left and right wheels. Furthermore, we will assume a pure rolling motion of the wheel with no slippage. Let $\alpha_{(\cdot)}$ be the rotation angle of the rear wheels. Under previous assumptions, the wheel dynamics can be described by $J_{w} \ddot{\alpha}_{(\cdot)}=\tau_{(\cdot)}$, where $J_{w}$ is the equivalent moment of inertia of the wheel and

$$
\tau_{(\cdot)}=\tau_{(\cdot)}^{h}+\tau_{(\cdot)}^{b}-b_{w} \dot{\alpha}_{(\cdot)},
$$

is the resulting torque acting on the wheel. It is a function of the thrust generated by the AP, who remains in charge of the motion, and of the applied brake torques. Specifically, $\tau_{(\cdot)}$ is given by the contribution of three terms. The torque $\tau_{(\cdot)}^{h}$ results from force exerted by the AP on the handles and transmitted to the wheel hub through the mechanical structure of walker. The second term is the applied braking torque $\tau_{(\cdot)}^{b}$. Finally, the term $-b_{w} \dot{\alpha}_{(\cdot)}$ accounts for the rolling resistance that opposes to the wheel rotation, where $b_{w}$ denotes the viscous friction coefficient around the wheel rotation axle. Similarly to [8], the braking action is modeled as a dissipative system, i.e.,

$$
\tau_{(\cdot)}^{b}=\left\{\begin{array}{cl}
-b_{(\cdot)}^{b} \dot{\alpha}_{(\cdot)} & \text { if } \dot{\alpha}_{(\cdot)} \neq 0 \\
c_{(\cdot)}^{b} \tau_{(\cdot)}^{h} & \text { if } \dot{\alpha}_{(\cdot)}=0
\end{array}\right.
$$

where $b_{(\cdot)}^{b} \in\left[0, b_{\max }\right]$ and $c_{(\cdot)}^{b} \in[0,1]$ are controllable variables determining the viscous frictions of the brakes. In case of servo brakes, coefficients $b_{(\cdot)}^{b}$ and $c_{(\cdot)}^{b}$ can be changed by varying the input current, thus allowing the control system to suitably modulate the braking torque [8].

\section{PRoblem Formulation}

If the cart were driven by two independent motors able to exert any left and right wheel torque $\tau_{l}$ and $\tau_{r}$, the control law described in the previous section could be easily implemented by computing the desired inputs $u_{f}$ and $u_{n}$ according to (7) and then inverting (5). However, the torques $\tau_{(\text {.) }}$ cannot be set arbitrarily since they depend on the human thrust $\tau_{(\cdot)}^{h}$ and the braking action $\tau_{(\cdot)}^{b}$, as expressed in (8). The braking torque $\tau_{(\cdot)}^{b}$, in turn, is subject to additional constraints as implied by model (9). For instance, the braking torque has always opposite sign of the angular wheel speed. In this respect, while the passive robotics approach brings in a number of benefits in terms of safety, weight and cost, it poses several challenges when it comes to designing a guidance system based solely on braking actions. The approach to this problem adopted in this work is to treat the control signals in (7) as reference values $u_{f}^{d}$ and $u_{n}^{d}$. Then, the braking torques $\tau_{(\cdot)}^{b}$ in (8) are modulated according to the model (9) so that the actual $u_{f}$ and $u_{n}$ resulting from (5) approximate the desired signals $u_{f}^{d}$ and $u_{n}^{d}$. In practice, since the AP is in charge of the motion of the walker, we are not interested in enforcing a specific forward speed profile $v_{d}$. Rather, we exploit this additional degree of freedom in order to minimize the magnitude of the corrective actions. This amounts to tracking only one of the two reference signals (e.g., $u_{n}^{d}$ ) while adjusting the other $\left(u_{f}\right)$ so as to optimize a suitable cost function accounting for AP's comfort. This leads to Optimal Control by Brakes (OCB) problem

$$
\begin{aligned}
\min _{\tau^{b}} & \left\|\tau^{b}\right\|_{1} \\
\text { s.t. } \quad-b_{\max } \operatorname{sgn}\left(\dot{\alpha}_{(\cdot)}\right) \dot{\alpha}_{(\cdot)} & \leq \operatorname{sgn}\left(\dot{\alpha}_{(\cdot)}\right) \tau_{(\cdot)}^{b} \leq 0, \\
\tau_{r}^{b}-\tau_{l}^{b} & =\tilde{u}_{n}^{d} b_{\max },
\end{aligned}
$$

where $\tau^{b}=\left[\tau_{l}^{b}, \tau_{r}^{b}\right]^{\prime}, \operatorname{sgn}(\cdot)$ denotes the sign function and the auxiliary torque reference is given by $\tilde{u}_{n}^{d}=\left(u_{n}^{d}-\tau_{r}^{h}+\right.$ $\left.\tau_{l}^{h}+b_{w}\left(\dot{\alpha}_{r}-\dot{\alpha}_{l}\right)\right) / b_{\max }$. In plain words, constraint (11) models the dissipative nature of the braking torques (9), while (12) ensures the tracking of the reference signal $u_{n}^{d}$. In fact, by substituting the definition of $\tilde{u}_{n}^{d}$ in (12) and exploiting (8) and (5), it can be easily verified that (12) corresponds to enforcing $\tau_{r}-\tau_{l}=u_{n}^{d}$. The cost function in (10) is an index accounting for human comfort. The intuition suggests that from the user's perspective, the smaller the correction made by the guidance system, the higher the comfort.

\section{A. Human Thrust Estimation Algorithm}

In order to compute the auxiliary torque reference $\tilde{u}_{n}^{d}$, the torques $\tau_{(\cdot)}^{h}$ resulting from the thrust of the AP have to be known. A possible approach, aiming at keeping the cost of the walker low, is to estimate the torques $\tau_{(\cdot)}^{h}$ and the wheel speeds $\dot{\alpha}_{(\cdot)}$ using data from accelerometers and encoders, filtered with a Kalman filter. Due to space limits, only a sketch of the approach is here proposed.

By knowing one wheel mechanical power $P_{(\cdot)}(t)=$ $\tau_{(\cdot)}(t) \dot{\alpha}_{(\cdot)}(t)$, it is possible to estimate the work of a wheel $W_{(\cdot)}\left(\Delta_{t}\right)$ in an interval $\Delta_{t}=t_{k+1}-t_{k}$ as

$$
W_{(\cdot)}\left(\Delta_{t}\right)=\int_{t_{k}}^{t_{k+1}} P_{(\cdot)}(t) d t=\frac{1}{2} J_{w}\left(\dot{\alpha}_{(\cdot)}\left(t_{k+1}\right)^{2}-\dot{\alpha}_{(\cdot)}\left(t_{k}\right)^{2}\right) .
$$

Hence, the mean power between $t_{k+1}$ and $t_{k}$

$$
\Delta_{t} P_{(\cdot)}\left(t_{k}\right)=W_{(\cdot)}\left(t_{k+1}\right)-W_{(\cdot)}\left(t_{k}\right) .
$$

Plugging (13) into (14), and defining $\varepsilon_{k}=\Delta_{t} \dot{\alpha}_{(\cdot)}\left(t_{k}\right)$ as the wheel encoder increment in the interval $t_{k}-t_{k-1}$, we get

$$
\tau_{(\cdot)}\left(t_{k}\right)=\frac{J_{w}}{2} \frac{\varepsilon_{k}^{2}-\varepsilon_{k-1}^{2}}{\Delta_{t}^{2} \varepsilon_{k}} .
$$

Using (8) and assuming the knowledge of the braking action, the previous relation yields to a relation between the encoder readings and the human applied torques $\tau_{(\cdot)}^{h}$. 


\section{STEERING BY BRAKES}

Due to the limitations on the possible braking torques that can be generated, the OCB problem may or may not admit a feasible solution, depending on the reference signal $u_{n}^{d}$ resulting from the control law (7). This may or may not result in a feasible reference signal $\tilde{u}_{n}^{d}$ to be tracked. In this section, we will first derive the closed form solution to OCB when a feasible solution does indeed exist. Then, we will discuss a possible strategy to cope with the case of unfeasible reference signal $\tilde{u}_{n}^{d}$. Finally, the it is shown how to modify the obtained solution in order to minimize the intrusiveness of the guidance system, while at the same time guaranteeing a desired level of safety.

\section{A. Feasible Reference $\tilde{u}_{n}^{d}$}

Denote by $\mathcal{N}$ the range of feasible reference signals $\tilde{u}_{n}^{d}$, i.e. such that (10)-(12) admits a feasible solution. Simple geometric arguments allows one to characterize $\mathcal{N}$ explicitly. If $\operatorname{sgn}\left(\dot{\alpha}_{r}\right)=\operatorname{sgn}\left(\dot{\alpha}_{l}\right)$, we have

$$
\mathcal{N}=\left\{\tilde{u}_{n}^{d}:-\operatorname{sgn}\left(\dot{\alpha}_{l}\right) \dot{\alpha}_{r} \leq \operatorname{sgn}\left(\dot{\alpha}_{l}\right) \tilde{u}_{n}^{d} \leq \operatorname{sgn}\left(\dot{\alpha}_{l}\right) \dot{\alpha}_{l}\right\},
$$

otherwise (i.e., $\operatorname{sgn}\left(\dot{\alpha}_{r}\right) \neq \operatorname{sgn}\left(\dot{\alpha}_{l}\right)$ )

$$
\mathcal{N}=\left\{\tilde{u}_{n}^{d}: 0 \leq \operatorname{sgn}\left(\dot{\alpha}_{l}\right) \tilde{u}_{n}^{d} \leq \operatorname{sgn}\left(\dot{\alpha}_{l}\right)\left(\dot{\alpha}_{l}-\dot{\alpha}_{r}\right)\right\} .
$$

Under the assumption that the feasible set is not empty, a solution to problem (10)-(12) is given by the following proposition.

Proposition 1: If $\tilde{u}_{n}^{d}$ in (12) is such that $\tilde{u}_{n}^{d} \in \mathcal{N}$, where $\mathcal{N}$ is given by (15)-(16), then $\tau^{b^{*}}$ is an optimal solution to problem (10)-(12) if and only if it satisfies the following conditions.

- If $\dot{\alpha}_{l}<0, \dot{\alpha}_{r}<0$ or $\dot{\alpha}_{l}>0, \dot{\alpha}_{r}>0$, then

$$
\tau^{b^{*}}=\frac{1}{2} q \tilde{u}_{n}^{d} b_{\max }
$$

where

$$
q= \begin{cases}{\left[\operatorname{sgn}\left(\tilde{u}_{n}^{d}\right)-1, \operatorname{sgn}\left(\tilde{u}_{n}^{d}\right)+1\right]^{\prime},} & \text { if } \dot{\alpha}_{l}<0 \\ {\left[-\operatorname{sgn}\left(\tilde{u}_{n}^{d}\right)-1,-\operatorname{sgn}\left(\tilde{u}_{n}^{d}\right)+1\right]^{\prime} .} & \text { if } \dot{\alpha}_{l}>0\end{cases}
$$

- If $\dot{\alpha}_{l}<0, \dot{\alpha}_{r}>0$ or $\dot{\alpha}_{l}>0, \dot{\alpha}_{r}<0$, then

$$
\tau^{b^{*}}=b_{\max }\left(\gamma q_{1}+(1-\gamma) q_{2}\right)
$$

for any $\gamma \in[0,1]$, where

$$
\begin{aligned}
& q_{1}= \begin{cases}{\left[-\tilde{u}_{n}^{d}-\dot{\alpha}_{r},-\dot{\alpha}_{r}\right]^{\prime},} & \text { if } \tilde{u}_{n}^{d} \leq u_{1} \\
{\left[0, \tilde{u}_{n}^{d}\right]^{\prime},} & \text { if } \tilde{u}_{n}^{d}>u_{1}\end{cases} \\
& q_{2}= \begin{cases}{\left[-\dot{\alpha}_{l}, \tilde{u}_{n}^{d}-\dot{\alpha}_{l}\right]^{\prime},} & \text { if } \tilde{u}_{n}^{d} \geq u_{2} \\
{\left[-\tilde{u}_{n}^{d}, 0\right]^{\prime},} & \text { if } \tilde{u}_{n}^{d}<u_{2}\end{cases}
\end{aligned}
$$

and $u_{1}=\max \left\{\dot{\alpha}_{l},-\dot{\alpha}_{r}\right\}, u_{2}=\min \left\{\dot{\alpha}_{l},-\dot{\alpha}_{r}\right\}$.

Proof: First, let us consider the case of wheel angular velocities having the same sign, e.g. assume $\dot{\alpha}_{(\cdot)}<0$, the case $\dot{\alpha}_{(\cdot)}>0$ being similar. In this case, the statement is proved if the optimal solution is

$$
\tau^{b^{*}}= \begin{cases}{\left[0, u_{n}^{d} b_{\max }\right]^{\prime},} & \text { if } \tilde{u}_{n}^{d} \geq 0 \\ {\left[-u_{n}^{d} b_{\max }, 0\right]^{\prime} .} & \text { if } \tilde{u}_{n}^{d}<0\end{cases}
$$

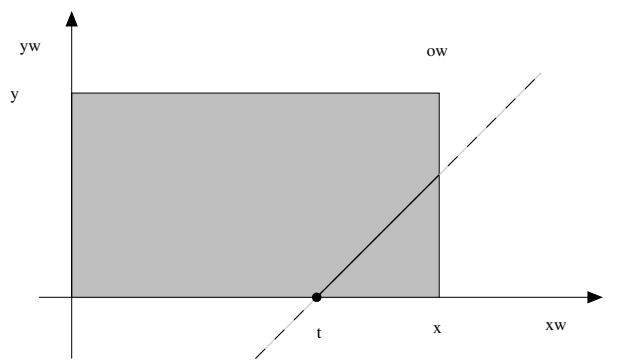

Fig. 3. Geometric representation of the OCB problem when $\dot{\alpha}_{l}<0$ and $\dot{\alpha}_{r}<0$ : admissible braking torque (dashed box), feasible set (solid segment) and optimal solution (dot).

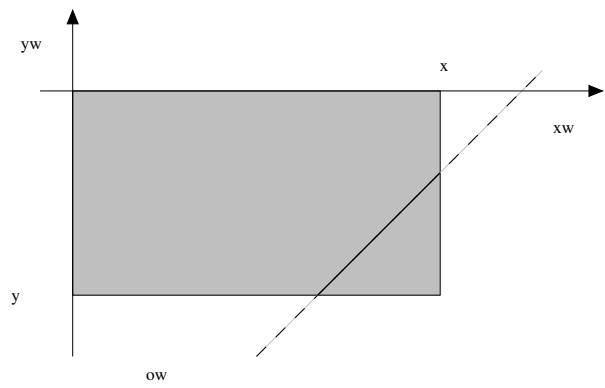

Fig. 4. Geometric representation of the OCB problem when $\dot{\alpha}_{l}<0$ and $\dot{\alpha}_{r}>0$ : admissible braking torque (dashed box), feasible set and optimal solutions (solid segment)

Since by hypothesis the reference torque $\tilde{u}_{n}^{d} \in \mathcal{N}$, the problem admits a solution and the feasible set is a segment, as shown in Fig. 3. Hence, the solution to (10)-(12) amounts to finding the point on such a segment which is closest (in the 1-norm) to the origin, from which the thesis readily follows.

Consider now the case of wheel angular velocities having different sign, and for the sake of exposition assume $\dot{\alpha}_{l}<0$ and $\dot{\alpha}_{r}>0$. Also in this case the feasible set is a non empty interval, as shown in Fig. 4. However, given the $45^{\circ}$ slope of such a segment, all its points lie at the same distance (in the 1-norm) to the origin. Hence, each feasible solution is an optimal one. Simple geometric arguments allow one to paremeterize the whole feasible set as written in the thesis.

Once the optimal braking torques $\tau^{b^{*}}(\cdot)$ have been computed, the corresponding braking commands $b_{(\cdot)}^{b}$ are derived by inverting (9).

\section{B. Unfeasible Reference $\tilde{u}_{n}^{d}$}

If the desired torque $u_{n}^{d}$ coming from the control law (7) translates in a reference signal $\tilde{u}_{n}^{d} \notin \mathcal{N}$, the problem does not admit a feasible solution. This means that the desired reference $\tilde{u}_{n}^{d}$ cannot be tracked by any braking torque. In this case, a possibility is to fully stop the cart for safety reason and then disengage the brakes after a given amount of time [11]. In this situation, audio/video interfaces, as well as wearable haptic devices, present on board the FriWalk, can be exploited to suggest the most appropriate direction of motion to the user. An alternative possibility to cope with an unfeasible reference signal $\tilde{u}_{n}^{d}$ is to modify the original OCB problem, by removing the equality constraint and looking 
for the brake torques resulting in a cart torque as close as possible to the desired one. This can be done by solving the optimization problem

$$
\begin{array}{ll}
\min _{\tau^{b}} & \left|\tau_{r}-\tau_{l}-\tilde{u}_{n}^{d} b_{\max }\right| \\
\text { s.t. } & -b_{\max } \operatorname{sgn}\left(\dot{\alpha}_{(\cdot)}\right) \dot{\alpha}_{(\cdot)} \leq \operatorname{sgn}\left(\dot{\alpha}_{(\cdot)}\right) \tau_{(\cdot)}^{b} \leq 0
\end{array}
$$

In this case, the feasible torque set specified by (18) is a box. Similar to what done in the proof of Proposition 1, geometric arguments show that the solution to problem (17)(18) corresponds to one of the vertices of the of the feasible set and can be computed in closed form. The proposed strategy yields a "best-effort" solution in the sense that does not guarantee the exact tracking of the desired torque $u_{n}^{d}$, but provides the best approximation compatible with the limited control authority provided by the braking system.

\section{Increasing the AP Comfort}

In order to minimize the intrusiveness of the guidance system, thus further increasing the human comfort, "small" deviations from the planned path may be tolerated. The underlying idea is to modulate the authority of the control system as a function of the tracking errors. A possible function weighting both the distance and orientation errors of the walker with respect to the desired path is

$$
V(l, \tilde{\theta})=\frac{1}{2}\left(l^{2}+\tilde{\theta}^{2}\right),
$$

which is a component of the Lyapunov function reported in (6). The guidance system exerts the maximum control authority, i.e., it enforces the exact braking torques resulting from the solution of OCB, only if $\mathbf{V}(l, \tilde{\theta}) \geq T_{d}$, with $T_{d}$ design parameter. Otherwise, the braking action is lowered proportionally to the value of $V(l, \tilde{\theta})$, being null when the tracking error is zero. By defining

$$
f(l, \tilde{\theta})=\min \left\{1, \frac{\mathbf{V}(l, \tilde{\theta})}{T_{d}}\right\},
$$

a Relaxed Optimal Control by Brakes (ROCB) problem can be formulated by replacing the equality constraint (12) with

$$
\tau_{r}^{b}-\tau_{l}^{b}=f(l, \tilde{\theta}) \tilde{u}_{n}^{d} b_{\max }
$$

Notice that, when $f(l, \tilde{\theta})=0$, no braking action is required. Conversely, if $f(l, \tilde{\theta})=1$, the original OCB problem is recovered. Clearly, the closed form solution provided by Proposition 1 still applies by simply replacing $\tilde{u}_{n}^{d}$ with $f(l, \tilde{\theta}) \tilde{u}_{n}^{d}$.

\section{NumericAl RESUlts}

Simulation results are reported in this section. Based on the current prototype of FriWalk, the wheels radius is set to $r=10[\mathrm{~cm}]$ while the wheel axle is set to $d=50[\mathrm{~cm}]$. Throughout this section, we will assume that the viscous friction of the wheels is $b_{w}=0.1[\mathrm{~N} \mathrm{~m} \mathrm{~s} / \mathrm{rad}]$, while the maximum viscous friction of the brakes $b_{\max }=0.1[\mathrm{~N} \mathrm{~m}$ $\mathrm{s} / \mathrm{rad}$ ] (see (8) and (9)). Moreover, the rolling resistance is also considered in the simulated wheel dynamic. Finally, we

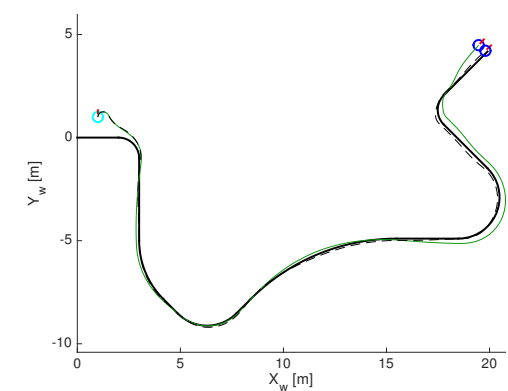

Fig. 5. Desired path (thick line), path followed by the cart with braking action with $T_{d}=0.5$ (thin line) and $T_{d}=0.02$ (thin dashed line).

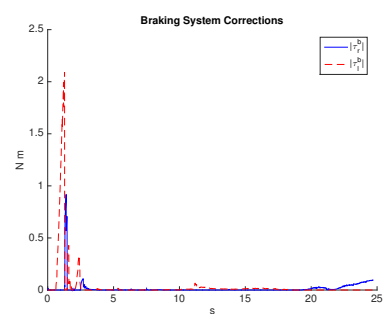

a)

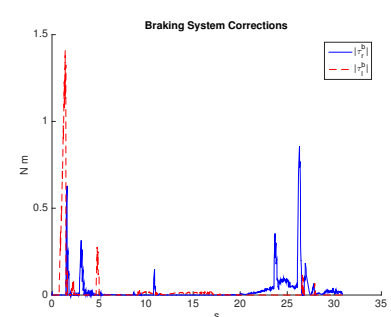

b)
Fig. 6. Braking action applied to the reference trajectory in Fig. 5 for $T_{d}=0.5$ (a) and $T_{d}=0.02(\mathrm{~b})$.

considered $\gamma=1$ as the tuning parameter of Proposition 1 and the modified solution presented in (17)-(18) is adopted for unfeasible torques.

In order to evaluate the effectiveness of the proposed solution, the forces that the user applies to the FriWalk during the motion have been synthetically generated. The desired path (thick line in Fig. 5) used for the simulations starts from $(0,0)$, while the vehicle initial configuration is in position $(1,1)$ with an orientation that is perpendicular to the path and pointing in the opposite direction. The algorithm is executed with a sampling time of 10 [ms], while the quantities of interest, i.e., the wheel velocities and torques, are estimated with a Kalman filter assuming noisy encoder measurements. In particular, the encoders are supposed to have a resolution of $10^{-2}$ [deg] and a noise of $\pm 10^{-2}$ [deg]. These figures have been estimated from the available prototype.

In the first simulation, the torques pushing the vehicle towards the path are generated with the dynamic controller obeying to (7). Nonetheless, two sinusoidal disturbances in quadrature, having amplitude of $10^{-1} \mathrm{Nm}$, have been added to such torque signals. Notice that, the guidance system in charge of controlling the braking system still obeys to (7) but with different parameters, hence enforcing the braking system to be active even in the absence of the sinusoidal disturbances. Fig. 5 reports with a thin line the path followed by the cart controlled by the braking system. Fig. 6-a reports the braking actions applied to the left and right wheels according to the proposed algorithm. Notice how at the beginning the braking action is more authoritative. Finally, Fig. 7-a reports the time evolution of the Lyapunov-like function describing the user comfort reported in (19). The 


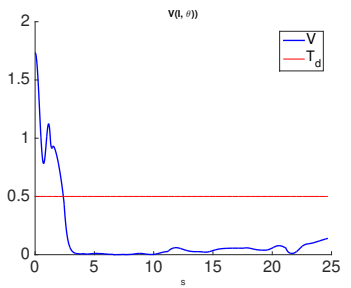

a)

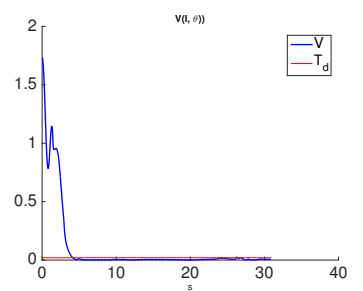

b)
Fig. 7. Lyapunov-like function for the reference trajectory in Fig. 5 when $T_{d}=0.5$ (a) and $T_{d}=0.02(\mathrm{~b})$.

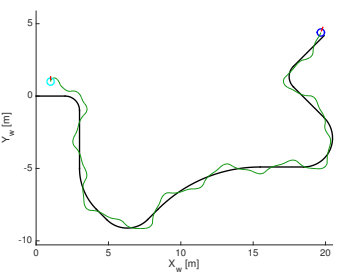

a)

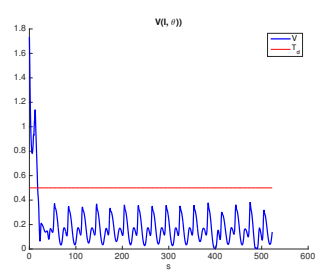

b)
Fig. 8. a) Desired path (thick line) and path followed by the cart with braking action with $T_{d}=0.5$ (thin line) when the cart is controlled with uncooperative torques. b) Associated Lyapunov-like function time behaviour for $T_{d}=0.5$.

system is controlled steadily below the chosen threshold of $T_{d}=0.5$. For comparison, Fig. 6-b and Fig. 7-b report the braking actions and the Lyapunov function when the threshold is lowered to applied to $T_{d}=0.02$. In this case, the braking action is more authoritative and, as a consequence, the trajectory is closer to the desired one (see Fig. 5, thin dashed line). Notice that for a tighter control, a more dissipative action is needed, hence the time to accomplish the full path grows from $25\left(T_{d}=0.5\right)$ to $32\left(T_{d}=0.02\right)$ seconds.

To prove the effectiveness of the algorithm for uncooperative behaviours, Fig. 8-a depicts the trajectory followed by the cart when the user constantly pushes the FriWalk away from the path, following a diverging sinusoidal reference torque. As visible from the results, the system is still able to control the FriWalk along the path, respecting the desired performance given by $T_{d}=0.5$ (Fig. $8-b$ ). However, the time the accomplish the path inevitably grows to more than 500 seconds.

\section{CONCLUSIONS}

In this paper, we have shown a guidance mechanism for a walking robotic assistant based on the use of differential braking. The proposed solution builds upon an available path following control law devised for the case of fully actuated carts and adapts it to meet the input constraints imposed by the braking-only guidance system. Our approach is based on the formulation of an optimisation problem and on its closedform solution and it strikes a good compromise between several and conflicting goals: 1. implementation on low cost devices, 2. minimal intrusiveness to preserve the user's comfort, 3. good levels of safety by the definition of a corridor that is never violated. The adopted solution, although clearly suboptimal in terms of tracking performance, turns out to be effective in gently guiding the user along desired paths, as confirmed by simulation results. An alternative approach would be to design the controller from scratch by explicitly considering the limited control authority due to the passive nature of the walker, in the same spirit of the recent work [17]. Our next goal in this research activity is to carry out a large scale validation with users with an implementation of the algorithm on the device. Such experimental campaign is expected to provide useful data in order to fine tune the cost function adopted to express the user comfort and to identify the best solution for an indirect estimate of the accelerations applied by the user without expensive sensors.

\section{REFERENCES}

[1] K.-T. Khaw, N. Wareham, S. Bingham, A. Welch, R. Luben, and N. Day, "Combined impact of health behaviours and mortality in men and women: The EPIC-Norfolk prospective population study," PLoS Med, vol. 5, no. 1, p. e12, 012008.

[2] "European Innovation Partnership on Active and Healthy Ageing," http://ec.europa.eu/active-healthy-ageing, 2015, [Mar 23, 2015].

[3] A. Goswami, M. Peshkin, and J. Colgate, "Passive robotics: An exploration of mechanical computation," in Proc. American Control Conference, 1990, pp. 2791-2796.

[4] M. Peshkin, J. Colgate, W. Wannasuphoprasit, C. Moore, R. Gillespie, and P. Akella, "Cobot architecture," IEEE Transactions on Robotics and Automation, vol. 17, no. 4, pp. 377-390, 2001.

[5] C. Huang, G. Wasson, M. Alwan, P. Sheth, and A. Ledoux, "Shared navigational control and user intent detection in an intelligent walker," in AAAI Fall 2005 Symposium, 2005.

[6] A. Rentschler, R. Cooper, B. Blasch, and M. Boninger, "Intelligent walkers for the elderly: Performance and safety testing of VAPAMAID robotic walker," Journal of rehabilitation research and development, vol. 40, no. 5, pp. 423-432, 2003.

[7] J. Frémy, F. Ferland, M. Lauria, and F. Michaud, "Force-guidance of a compliant omnidirectional non-holonomic platform," Robotics and Autonomous Systems, vol. 62, no. 4, pp. 579 - 590, 2014.

[8] Y. Hirata, A. Hara, and K. Kosuge, "Motion control of passive intelligent walker using servo brakes," IEEE Transactions on Robotics, vol. 23, no. 5, pp. 981-990, 2007.

[9] M. Saida, Y. Hirata, and K. Kosuge, "Development of passive type double wheel caster unit based on analysis of feasible braking force and moment set," in Proc. IEEE Int. Conf. on Intelligent Robots and Systems, 2011, pp. 311-317.

[10] C.-H. Ko, K.-Y. Young, Y.-C. Huang, and S. K. Agrawal, "Walk-assist robot: A novel approach to gain selection of a braking controller using differential flatness," IEEE Transactions on Control Systems Technology, vol. 21, no. 6, pp. 2299-2305, 2013.

[11] D. Fontanelli, A. Giannitrapani, L. Palopoli, and D. Prattichizzo, "Unicycle Steering by Brakes: a Passive Guidance Support for an Assistive Cart," in Proc. IEEE Int. Conf. on Decision and Control, 10-13 Dec. 2013, pp. 2275-2280.

[12] "ACANTO: A CyberphysicAl social NeTwOrk using robot friends," http://www.ict-acanto.eu/acanto, February 2015, EU Project.

[13] A. Colombo, D. Fontanelli, A. Legay, L. Palopoli, and S. Sedwards, "Motion Planning in Crowds using Statistical Model Checking to Enhance the Social Force Model," in Proc. IEEE Int. Conf. on Decision and Control. Florence, Italy: IEEE, 10-13 Dec. 2013, pp. 3602-3608.

[14] F. Barbagli, A. Formaglio, M. Franzini, A. Giannitrapani, and D. Prattichizzo, "An experimental study of the limitations of mobile haptic interfaces," Experimental Robotics IX, pp. 533-542, 2006. 
[15] A. Formaglio, D. Prattichizzo, F. Barbagli, and A. Giannitrapani, "Dynamic performance of mobile haptic interfaces," IEEE Transactions on Robotics, vol. 24, no. 3, pp. 559-575, 2008.

[16] D. Soetanto, L. Lapierre, and A. Pascoal, "Adaptive, non-singular pathfollowing control of dynamic wheeled robots," in Proc. IEEE Int. Conf. on Decision and Control, 2003, pp. 1765-1770.

[17] W. Wolfslag, M. Plooij, W. Caarls, S. van Weperen, and G. Lopes, "Dissipatively actuated manipulation," Control Engineering Practice, vol. 34, pp. 68 - 76, 2015. 